\title{
Quorum sensing in Staphylococcus infections
}

\author{
Jeremy M. Yarwood ${ }^{1}$ and Patrick M. Schlievert ${ }^{2}$ \\ ${ }^{1}$ Department of Microbiology, University of Iowa Roy J. and Lucille A. Carver College of Medicine, Iowa City, Iowa, USA \\ ${ }^{2}$ Department of Microbiology, University of Minnesota Medical School, Minneapolis, Minnesota, USA
}

\begin{abstract}
Quorum sensing via the accessory gene regulator $(a g r)$ system has been assigned a central role in the pathogenesis of staphylococci, particularly Staphylococcus aureus. While the control of virulence gene expression in vitro by agr has been relatively straightforward to describe, regulation of both the quorum response itself and virulence genes in vivo is considerably more complex. The quorum response is highly dependent upon the environment in which the organism is grown and is strongly influenced by additional regulators that respond to signals other than cell density. There is increasing evidence that the agr phenotype may influence the behavior and pathogenesis of biofilm-associated S. aureus and S. epidermidis and may contribute to the chronic nature of some biofilm-associated infections.
\end{abstract}

J. Clin. Invest. 112:1620-1625 (2003). doi:10.1172/JCI200320442.

Staphylococcus aureus and Staphylococcus epidermidis are Gram-positive cocci that normally colonize the epithelial surfaces of large numbers of humans (reviewed in ref. 1). S. epidermidis is considered part of the of the normal human microbial flora, while $S$. aureus is usually regarded as a transient member. Colonization by either species usually does not lead to adverse events. However, when these organisms or their extracellular products are allowed to breach the epithelial layer, serious disease can result (1). S. aureus has many cell surface virulence factors (such as protein A and clumping factor) and secreted exotoxins and enzymes that allow strains to cause a myriad of infections. These diseases range from relatively benign furuncles and subcutaneous abscesses to scalded skin syndrome, sepsis, necrotizing pneumonia, and toxic shock syndrome (TSS). While no single cell surface virulence factor has been shown to be uniquely required for mucous membrane attachment, once colonization occurs, numerous secreted exotoxins, including the pyrogenic toxin superantigens and exfoliative toxins, definitively cause serious human disease. Other secreted exotoxins, such as the four hemolysins $(\alpha, \beta, \delta$, and $\gamma)$ and Panton-Valentine leukocidin have also been suggested to contribute to signif-

\footnotetext{
Address correspondence to: Jeremy M. Yarwood, Department of Microbiology, University of Iowa Roy J. and Lucille A. Carver College of Medicine, 540 Eckstein Medical Research Building, 500 Newton Road, Iowa City, Iowa 52242, USA.

Phone: (319) 335-7996; Fax: (319) 335-7949;

E-mail: jeremy-yarwood@uiowa.edu.

Conflict of interest: The authors have declared that no conflict of interest exists.

Nonstandard abbreviations used: toxic shock syndrome (TSS); accessory gene regulator $(a g r)$; autoinducing peptide (AIP); staphylococcal accessory regulator (SarA); repressor of toxins (Rot); polysaccharide intercellular adhesin (PIA).
}

icant illnesses. S. epidermidis does not possess the array of extracellular toxins that $S$. aureus does, and its primary virulence factor is considered to be its ability to form biofilms $(2,3)$.

\section{The accessory gene regulator quorum sensing system}

To improve their ability to cause this variety of human disease and to occupy numerous niches within the host, staphylococci have developed quorum-sensing systems that enable cell-to-cell communication and regulation of numerous colonization and virulence factors. The staphylococcal accessory gene regulator (agr) quorumsensing system decreases the expression of several cell surface proteins and increases the expression of many secreted virulence factors in the transition from lateexponential growth to stationary phase in vitro $(4,5)$. Expression of agr was found to contribute to staphylococcal pathogenesis in several infection models, including murine subcutaneous abscesses (6) and arthritis (7), as well as rabbit endocarditis (8). Expression of agr also appears to be involved in the invasion and apoptosis of epithelial cells (9). Interestingly, different agr groups, as defined by their production and recognition of distinct secreted signals, are associated predominantly with certain diseases (5). The reasons for this association between agr group and infection type are not yet clear, but a better understanding of this phenomenon may contribute to our understanding of the epidemiology of staphylococcal diseases.

Two primary transcripts, RNAII and RNAIII, are generated by the agr locus and originate from the $\mathrm{P} 2$ and $\mathrm{P} 3$ promoters, respectively (Figure 1). The P2 operon encodes four proteins that generate the agr-sensing mechanism. AgrB is a transmembrane protein that 


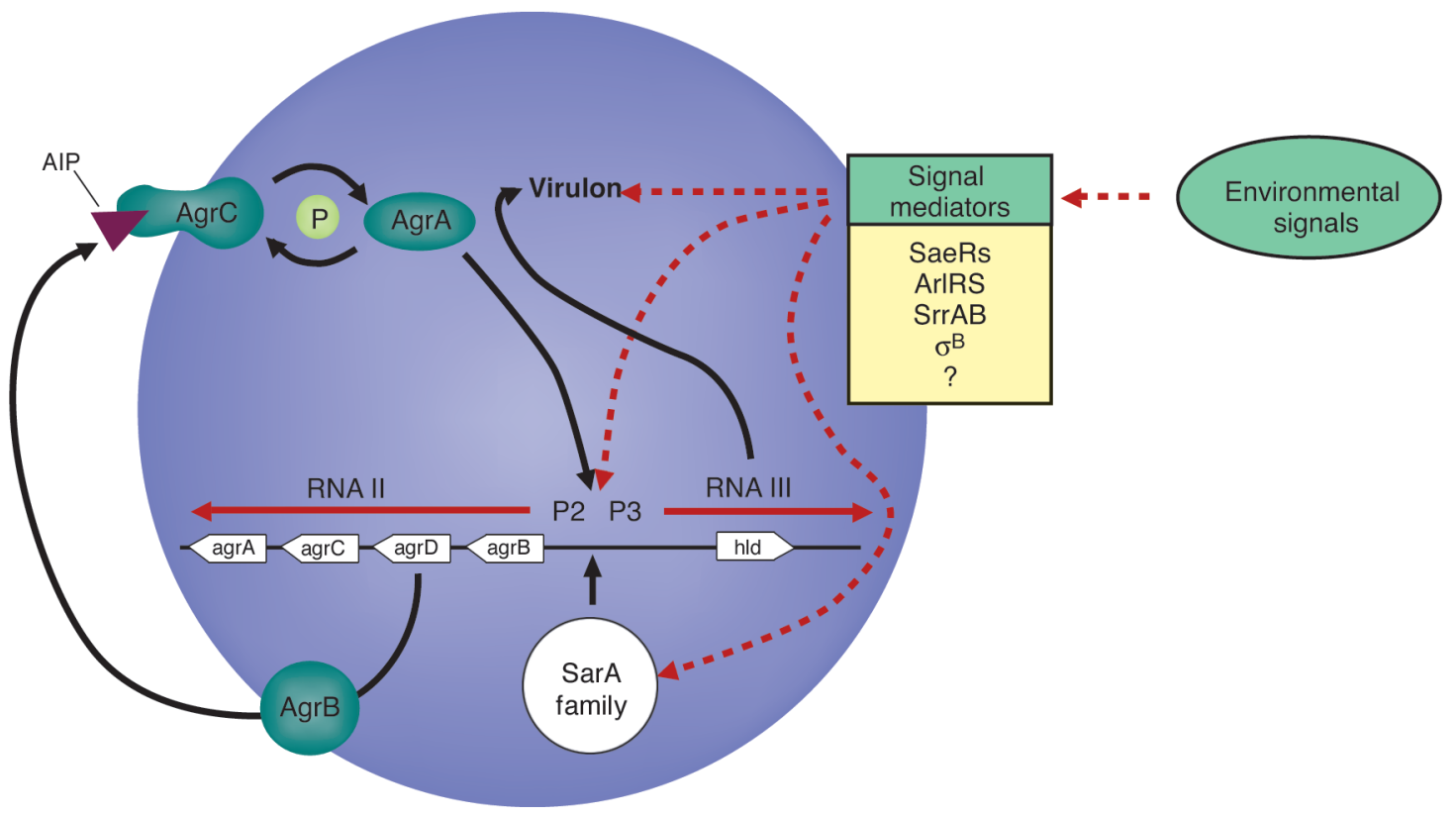

Figure 1

The accessory gene regulator (agr) system in Staphylococcus. The P2 operon encodes (via RNAll) the signaling mechanism, whereas the transcript of the P3 operon, RNAIII, acts as the effector molecule of the agr locus. Additional regulators of the quorum response and virulence genes, described in the text, are listed. Potential regulatory pathways used by environmental signals are indicated by the dashed red lines.

appears to be involved in (a) processing of the $\operatorname{agrD}$ product into an octapeptide; (b) secretion of the autoinducing peptide (AIP) signal; and (c) modification of the AIP by the formation of a cyclic thiolactone bond between an internal cysteine and the carboxyl terminus. AgrA and AgrC form a two-component regulatory system in which the transmembrane component, AgrC (histidine kinase), binds the extracellular AIP and in turn modulates the activity of AgrA, the response regulator. Through an as-yet-undefined mechanism, AgrA activity then leads to greatly increased P2 and P3 transcription in the late-log phase of growth, when the concentration of the signal in the medium is high. Sequence variation in $\operatorname{agr} B, \operatorname{agr} D$, and $\operatorname{agr} C$ has led to the identification of at least four $S$. aureus agr specificity groups in which AIP produced by one group inhibits agr expression in other groups (5).

Increased transcription of the $\mathrm{P} 3$ operon results in dramatically increased levels of intracellular RNAIII. RNAIII encodes the toxin $\delta$-hemolysin (via bld) but, more importantly, increases the transcription (and in some cases, translation) of several secreted virulence factors, including TSS toxin-1 and other hemolysins. However, some toxins, such as enterotoxins $\mathrm{A}$ and $\mathrm{K}$, made typically in low concentrations and during exponential phase, are not regulated by RNAIII. Other secreted toxins, such as enterotoxins $\mathrm{B}, \mathrm{C}$, and $\mathrm{D}$, are only partially upregulated by RNAIII and can be made in high concentrations independently of agr. At the same time, the expression of several cell surface virulence factors is decreased. It is easy to imagine the role that such coordination of virulence gene expression might play in certain infections, such as the formation of a walled-off furuncle. Initially, the staphylococci, present in small numbers, express their cell surface virulence factors in order to evade the host immune system. For example, protein A binds the Fc portion of IgG, and clumping factor may help to form the walledoff infection site. When this site becomes depleted of nutrients due to increased bacterial numbers, the organisms increase secreted factor production, allowing the organisms to escape the walled-off site and spread through the host tissues.

The potential role of agr-mediated quorum sensing is not as clear in other host environments. We and other researchers have shown through in vivo human and animal studies that agr appears to be unnecessary in certain infections for the expression of secreted virulence factors $(5,10-12)$. For example, agr expression in a rabbit abscess model was decreased at the same time that the animals developed TSS through exotoxin production (10). In addition, an agr mutant was just as effective at causing TSS as the isogenic wild-type organism. These studies do not rule out roles for agr in other aspects of these diseases, as will be discussed later in this review. But the studies do suggest that additional regulatory mechanisms are integral in regulation of both the quorum response and overall virulence of staphylococci.

\section{Additional regulators of the quorum response}

The agr quorum-sensing system has historically been assigned a central role in the model of $S$. aureus pathogenesis. Thus, studies of other known regulators of staphylococcal virulence have usually examined their 
interaction with the agr system. These additional regulators allow the organism to respond to environmental signals in addition to bacterial cell density, and sometimes counter agr activity (reviewed in ref. 5).

Among these regulators are the two-component systems (a family that includes AgrAC) that allow staphylococci to sense and respond to various environmental stimuli. SaeRS was the second two-component system involved in global regulation of virulence factors to be identified $(13,14)$ after AgrAC. sae mutants produce substantially less hemolysin and coagulase but have no effect on the production of RNAIII. However, the expression of at least one sae transcript was decreased in an agr mutant, suggesting that the sae system acts downstream of $a g r(15)$. It has been proposed, but not confirmed, that the sae locus responds to several environmental stimuli, including high salt, low $\mathrm{pH}$, glucose, and subinhibitory concentrations of antibiotics (5).

ArlRS comprises a third two-component system that appears to counter agr autoinduction by repressing production of hemolysins and exoenzymes $(16,17)$. Expression of arlRS was itself reduced in an $a g r$ mutant. An arls mutant was enhanced for biofilm formation, despite the increased expression of agr and the presumed downregulation of surface-associated adhesion factors. ArlRS also appears to regulate autolytic activity as well as the multidrug efflux pump NorA of $S$. aureus. These data suggest that under certain conditions, agr activity may influence the resistance of $S$. aureus to antibiotics.

A fourth two-component system, SrrAB, recently identified by our group (18) and others (19), was found to inhibit RNAIII expression and may itself be repressed by agr. Mutants of $\operatorname{srr} A B$ are unable to grow normally under anaerobic conditions $(18,19)$, and expression of $\operatorname{sr} A B$ was shown to regulate genes involved in energy metabolism (19). The signal for the system may be menaquinone, an intermediate in the oxidative respiratory pathway. Thus, SrrAB may be one link between energy metabolism in the cell and the quorum response.

The second major family of regulators of staphylococcal virulence are the DNA-binding proteins, including staphylococcal accessory regulator (SarA) and its homologs (reviewed in ref. 20). SarA, transcribed from three promoters within the same locus, was reported to be required for full agr transcription (21). In several reports, SarA has been shown to affect the expression of a wide array of virulence genes, sometimes acting independently of agr to decrease the expression of several exoproteins. SarA is responsive to some environmental conditions through intermediate regulators (22) and likely affects expression of the agr locus accordingly.

Additional transcription regulators, whose interactions with the agr system deserve further investigation, are the repressor of toxins (Rot) and the alternative sigma factor B. Both of these have been shown to affect the expression of numerous virulence-associated genes. Rot appears to counter agr activity, and a mutation in rot was shown to partially restore the wild-type phenotype of an agr mutant $(23,24)$. Sigma factor B, which responds to environmental stress (25), also appears to at times counter agr activity in that it increases the expression of some exoproteins early in growth (26).

Thus, the quorum response in staphylococci during infection occurs within the context of a complex regulatory network that continually modifies either $a g r$ activity itself or its downstream effects. Additional monitoring of gene expression and protein profiles in vivo will be required to understand this regulatory network that likely differs substantially from what has been described thus far in vitro.

\section{Quorum sensing and staphylococcal biofilms}

Many infections by staphylococci are not caused by the free-living organism but rather by groups of interacting cells termed biofilms. Bacterial biofilms are broadly defined as a community of cells attached to either an abiotic or a biotic surface, are encased in a self-produced matrix, and generally exhibit an altered growth and gene expression profile compared with that of planktonic, or free-living, bacteria (reviewed in ref. 27). Biofilm-associated infections have special clinical relevance, as they are generally resistant to antibiotic therapy and clearance by host defenses. In staphylococcal infections, these diseases include endocarditis $(8,28)$, osteomyelitis $(29,30)$, implanted device-related infections (27), and even some skin infections (31).

Two stages of staphylococcal biofilm formation have been described (reviewed in ref. 3). The first stage involves attachment of cells to a surface. This stage of biofilm formation is likely to be mediated in part by cell wall-associated adhesins, including the microbial surface components recognizing adhesive matrix molecules (MSCRAMMs). The second stage of biofilm development includes cell multiplication and formation of a mature, multi-layered, structured community. This stage is associated with production of extracellular factors, including the polysaccharide intercellular adhesin (PIA) component of the extracellular matrix. Detachment of cells from the established biofilm may then allow staphylococci to spread and colonize new sites. One of the most intriguing areas of investigation is determining what impact quorum sensing has on the growth, development, and pathogenesis of staphylococcal biofilms. There is mounting evidence that the agr phenotype and expression patterns may influence several aspects of biofilm behavior, including attachment of cells to surfaces, biofilm dispersal, and even the chronic nature of many biofilm-associated infections. Indeed, many of the products involved in biofilm development, including $\alpha$-toxin, surface-associated adhesins, $\delta$-hemolysin, and the autolysin AtlE (in S. epidermidis), are regulated by the agr system, at least in vitro. Furthermore, quorum sensing has been shown to be involved in biofilm development of several Gram-positive and Gram-negative bacteria, including Streptococcus mutans (32) and Pseudomonas aeruginosa (33). 
The limited number of studies addressing the quorum response and staphylococcal biofilms appear at first glance to be somewhat conflicting in their results and interpretation. Pratten et al. (34) found little difference between wild-type $S$. aureus and an agr mutant in adherence to either uncoated or fibronectin-coated glass under flow conditions, even though bld was expressed. In another study, RNAIII expression decreased $S$. aureus adherence to fibrinogen under static conditions, but increased adherence to fibronectin and human endothelial cells in both static and flow conditions (35). Vuong et al. (36) found that those $S$. aureus strains with a nonfunctional agr were much more likely to form biofilms under static conditions. $\alpha$-toxin, positively regulated by the agr system, was recently shown to be required for biofilm formation under both static and flow conditions (37). In an experimental endocarditis study, RNAIII expression increased with increasing $S$. aureus densities in vegetations (38), confirming the cell density-dependent expression of RNAIII in vivo. Interestingly, expression of RNAIII also occurred through a mechanism independent of the AgrAC signaling system, suggesting that there are additional, unidentified in vivo signals that regulate the quorum response. Taken together, these studies indicate that the precise role of $\mathrm{agr}$ expression in biofilm development is dependent upon the conditions in which the biofilm is grown, and suggest that differences in $S$. aureus strains may also introduce variability into the results.

Most infections of indwelling medical devices are caused by $S$. epidermidis, an organism with few exotoxins and for which the ability to form biofilms is considered the primary virulence factor (2). Recently, Vuong et al. (39) found that disruption of the agr locus in S. epidermidis resulted in increased attachment of the bacteria to polystyrene, increased biofilm formation, and higher expression of AtlE, which enhances attachment to abiotic surfaces. They also confirmed that the clinical isolate S. epidermidis O-47, the strain of choice for studying biofilm formation in S. epidermidis, was an agr mutant. Interestingly, agr did not regulate PIA expression.

Even in conditions in which agr does not appear to contribute to biofilm growth or development, it may still affect the virulence of biofilm-associated bacteria. We have observed expression of the agr system in conditions in which agr did not appear to affect biofilm growth or structure (40). It has been proposed that the production of $\delta$-hemolysin, a molecule with surfactant properties that is encoded by the agr locus, may contribute to the detachment of cells from both $S$. aureus and $S$. epidermidis biofilms $(36,39)$. Should this in fact be the case, it has important clinical implications. Cells expressing agr and actively detaching from the biofilm not only may establish additional infection sites in the host but also may contribute to the toxemia associated with acute staphylococcal infections. These cells are likely to express secreted virulence factors, including the superantigenic toxins of $S$. aureus. On the other hand, cells that remain in the biofilm and do not express agr may contribute to persistent, low-level infections, particularly in the case of S. epidermidis.

Evidence of selection for an agr-negative phenotype in chronic infections is emerging. Schwan et al. (41) studied chronic wound infections using a murine abscess model. After establishing infections with a hemolytic (thus with a presumably functional agr locus) $S$. aureus strain, the number of nonhemolytic bacteria recovered from the wounds increased over time. The authors suggest that several of the nonhemolytic isolates had mutations in the agr locus, although this was not directly shown. In mixed-strain infection experiments using normal, hyperhemolytic, and nonhemolytic strains to inoculate the mouse, the population of hyperhemolytic isolates declined (44.0-9.3\%) after 7 days, while the nonhemolytic group (presumably agr defective) increased (23.7-61.0\%) over the same period of time. Conversely, in both this study and several previous ones, infections established with single strains resulted in decreased cell numbers recovered from the infection of the agr mutant compared with those of the wild-type strain. Thus, in the mixedstrain infection experiments, functions performed by the wild-type strain may assist the nonhemolytic group in establishing infection. Also consistent with a selection for an agr-negative phenotype, expression of virulence factors in epidemic methicillin-resistant strains is shifted away from extracellular toxins and enzymes toward expression of surface proteins and colonization factors (42). Furthermore, agr mutants can frequently be found in isolates from clinical settings $(36,39)$ and arise spontaneously in vitro (43). When Vuong et al. (36) examined the correlation between a functional agr system and the ability of $S$. aureus clinical isolates to adhere to polystyrene under static conditions, they found that only $6 \%$ of the isolates with a functional agr system formed a biofilm in these conditions, compared with $78 \%$ of the agr-defective isolates. Failure of the strains with functional agr loci to form a biofilm was thought to be due in part to the surfactant properties of the $\delta$-hemolysin produced by these strains. These studies are all consistent with the idea that whereas secreted virulence factors may be important during the acute phase of infection, loss of agr function may enhance the long-term survival of staphylococci in the host and contribute to persistent (often biofilm-associated) infections. The enhanced survival of agr mutants might be due in part to the decreased production of immunostimulatory factors, such as superantigens, and increased expression of immune-evading factors, such as protein A.

It has been proposed that use of agr-inhibiting substances, such as AIP produced by staphylococci belonging to a different agr group, might be beneficial in the treatment of acute staphylococcal infection (5). Such treatments may decrease the production of extracellular virulence factors normally upregulated by agr expression. However, because loss of agr activity nor- 
mally correlates with increased expression of adhesin factors and decreased expression of potential dispersion factors, inhibition of agr activity may instead result in the conversion of an acute infection into a chronic one, particularly in biofilm-associated infections. Indeed, inhibition of agr activity increases attachment of both S. aureus and S. epidermidis to polystyrene (36, 39 ) and enhances biofilm formation. Furthermore, in certain animal models of infection, expression of agr does not significantly affect the expression of virulence factors, as would be expected from in vitro data (10-12). Understanding these caveats, additional investigation into agr inhibition is still warranted, given that inhibition of agr activity, and thus extracellular toxin and enzyme production, may be beneficial in some acute infection types.

Taken as a whole, the literature suggests that the role of quorum sensing in staphylococcal infections may not always be immediately obvious, as it varies with infection type, host environment, and even time. For example, in models in which agr does not appear to strongly influence virulence, its expression (or lack thereof) may contribute to biofilm formation. Conversely, in models in which agr expression does not appear to affect biofilm formation, it may still regulate virulence factor production. This makes the development of relevant in vitro and in vivo models a challenging proposition. Yet it is critical that further investigation of this area take place. The expression of the agr quorum-sensing system, already known to affect virulence factor production, may also affect everything from antibiotic resistance to energy metabolism through its interaction with other staphylococcal gene regulators. Furthermore, the agr phenotype and expression patterns in both $S$. aureus and S. epidermidis biofilms may influence when these infections become chronic or acute. Future studies should include sampling of staphylococcal infections for agr phenotype, evaluation of agr expression patterns in vivo using animal models of infection, and further investigation of agr function in biofilm growth and development.

\section{Acknowledgments}

J.M. Yarwood is supported by a Ruth M. Kirchstein National Research Service Award from the National Institutes of Health (GM069110). We thank Debra Murray for critical reading of the manuscript.

1. Tenover, F.C., and Gaynes, R.P. 2000. The epidemiology of Staphylococcus aureus infections. In Gram-positive pathogens. V.A. Fischetti, R.P. Novick, J.J. Ferretti, D.A. Portnoy, and J.I. Rood, editors. ASM Press. Washington, DC, USA. 414-421.

2. Raad, I., Alrahwan, A., and Rolston, K. 1998. Staphylococcus epidermidis: emerging resistance and need for alternative agents. Clin. Infect. Dis. 26:1182-1187.

3. Gotz, F. 2002. Staphylococcus and biofilms. Mol. Microbiol. 43:1367-1378.

4. Vuong, C., Gotz, F., and Otto, M. 2000. Construction and characterization of an agr deletion mutant of Staphylococcus epidermidis. Infect. Immun. 68:1048-1053.

5. Novick, R.P. 2003. Autoinduction and signal transduction in the regulation of staphylococcal virulence. Mol. Microbiol. 48:1429-1449.

6. Bunce, C., Wheeler, L., Reed, G., Musser, J., and Barg, N. 1992. Murine model of cutaneous infection with gram-positive cocci. Infect. Immun. 60:2636-2640.
7. Abdelinour, A., Arvidson, S., Bremell, T., Ryden, C., and Tarkowski, A 1993. The accessory gene regulator (agr) controls Staphylococcus aureus virulence in a murine arthritis model. Infect. Immun. 61:3879-3885.

8. Cheung, A.L., et al. 1994. Diminished virulence of a sar /agr mutant of Staphylococcus aureus in the rabbit model of endocarditis. J. Clin. Invest. 94:1815-1822.

9. Wesson, C.A., et al. 1998. Staphylococcus aureus Agr and Sar global regulators influence internalization and induction of apoptosis. Infect. Immun. 66:5238-5243

10. Yarwood, J.M., McCormick, J.K., Paustian, M.L., Kapur, V., and Schlievert, P.M. 2002. Repression of the Staphylococcus aureus accessory gene regulator in serum and in vivo. J. Bacteriol. 184:1095-1101.

11. Goerke, C., et al. 2000. Direct quantitative transcript analysis of the agr regulon of Staphylococcus aureus during human infection in comparison to the expression profile in vitro. Infect. Immun. 68:1304-1311.

12. Goerke, C., Fluckiger, U., Steinhuber, A., Zimmerli, W., and Wolz, C. 2001. Impact of the regulatory loci $a g r$, sarA and sae of Staphylococcus aureus on the induction of $\alpha$-toxin during device-related infection resolved by direct quantitative transcript analysis. Mol. Microbiol. 40:1439-1447.

13. Giraudo, A.T., Cheung, A.L., and Nagel, R. 1997. The sae locus of Staphylococcus aureus controls exoprotein synthesis at the transcriptional level. Arch. Microbiol. 168:53-58.

14. Giraudo, A.T., Calzolari, A., Cataldi, A.A., Bogni, C., and Nagel, R. 1999. The sae locus of Staphylococcus aureus encodes a two-component regulatory system. FEMS Microbiol. Lett. 177:15-22.

15. Giraudo, A.T., Mansilla, C., Chan, A., Raspanti, C., and Nagel, R. 2003. Studies on the expression of regulatory locus sae in Staphylococcus aureus. Curr. Microbiol. 46:246-250.

16. Fournier, B., and Hooper, D.C. 2000. A new two-component regulatory system involved in adhesion, autolysis, and extracellular proteolytic activity of Staphylococcus aureus. J. Bacteriol. 182:3955-3964.

17. Fournier, B., Klier, A., and Rapoport, G. 2001. The two-component system ArlS-ArlR is a regulator of virulence gene expression in Stapbylococcus aureus. Mol. Microbiol. 41:247-261.

18. Yarwood, J.M., McCormick, J.K., and Schlievert, P.M. 2001. Identification of a novel two-component regulatory system that acts in global regulation of virulence factors of Staphylococcus aureus. J. Bacteriol. 183:1113-1123.

19. Throup, J.P., et al. 2001. The srbSR gene pair from Staphylococcus aureus: genomic and proteomic approaches to the identification and characterization of gene function. Biochemistry. 40:10392-10401.

20. Cheung, A.L., and Zhang, G. 2002. Global regulation of virulence determinants in Staphylococcus aureus by the SarA protein family. Front. Biosci. 7:1825-1842.

21. Cheung, A.L., Bayer, M.G., and Heinrichs, J.H. 1997. sar genetic determinants necessary for transcription of RNAII and RNAIII in the agr locus of Staphylococcus aureus. J. Bacteriol. 179:3963-3971.

22. Deora, R., Tseng, T., and Misra, T. 1997. Alternative transcription factor $\sigma^{\mathrm{SB}}$ of Staphylococcus aureus: characterization and role in transcription of the global regulatory locus sar. J. Bacteriol. 179:6355-6359.

23. McNamara, P.J., Milligan-Monroe, K.C., Khalili, S., and Proctor, R.A. 2000. Identification, cloning, and initial characterization of rot, a locus encoding a regulator of virulence factor expression in Staphylococcus aureus. J. Bacteriol. 182:3197-3203.

24. Said-Salim, B., et al. 2003. Global regulation of Staphylococcus aureus genes by Rot. J. Bacteriol. 185:610-619.

25. Chan, P.F., Foster, S.J., Ingham, E., and Clements, M.O. 1998. The Staphylococcus aureus alternative sigma factor $\sigma^{\mathrm{B}}$ controls the environmental stress response but not starvation survival or pathogenicity in a mouse abscess model. J. Bacteriol. 180:6082-6089.

26. Nicholas, R.O., et al. 1999. Isolation and characterization of a sigB deletion mutant of Staphylococcus aureus. Infect. Immun. 67:3667-3669.

27. Donlan, R.M., and Costerton, J.W. 2002. Biofilms: survival mechanisms of clinically relevant microorganisms. Clin. Microbiol. Rev. 15:167-193.

28. van Wamel, W., et al. 2002. Regulation of Staphylococcus aureus type 5 capsular polysaccharides by agr and sarA in vitro and in an experimental endocarditis model. Microb. Pathog. 33:73-79.

29. Gillaspy, A., et al. 1995. Role of the accessory gene regulator $(a g r)$ in pathogenesis of staphylococcal osteomyelitis. Infect. Immun. 63:3373-3380.

30. Blevins, J.S., et al. 2003. Role of sarA in the pathogenesis of Staphylococcus aureus musculoskeletal infection. Infect. Immun. 71:516-523.

31. Akiyama, H., et al. 2003. Confocal laser scanning microscopic observation of glycocalyx production by Staphylococcus aureus in skin lesions of bullous impetigo, atopic dermatitis and pemphigus foliaceus. Brit. J. Dermatol. 148:526-532.

32. Li, Y.H., et al. 2002. A quorum-sensing signaling system essential for genetic competence in Streptococcus mutans is involved in biofilm formation. J. Bacteriol. 184:2699-2708.

33. Davies, D.G., et al. 1998. The involvement of cell-to-cell signals in the development of a bacterial biofilm. Science. 280:295-298. 
34. Pratten, J., Foster, S.J., Chan, P.F., Wilson, M., and Nair, S.P. 2001. Staphylococcus aureus accessory regulators: expression within biofilms and effect on adhesion. Microbes Infect. 3:633-637.

35. Shenkman, B., et al. 2002. Role of agr (RNAIII) in Staphylococcus aureus adherence to fibrinogen, fibronectin, platelets and endothelial cells under static and flow conditions. J Med. Microbiol. 51:747-754.

36. Vuong, C., Saenz, H.L., Gotz, F., and Otto, M. 2000. Impact of the agr quorum-sensing system on adherence to polystyrene in Staphylococcus aureus. J. Infect. Dis. 182:1688-1693.

37. Caiazza, N.C., and O'Toole, G.A. 2003. Alpha-toxin is required for biofilm formation by Staphylococcus aureus. J. Bacteriol. 185:3214-3217.

38. Xiong, Y.Q., et al. 2002. Activation and transcriptional interaction between agr RNAII and RNAIII in Staphylococcus aureus in vitro and in an experimental endocarditis model. J. Infect. Dis. 186:668-677.

39. Vuong, C., Gerke, C., Somerville, G.A., Fischer, E.R., and Otto, M. 2003 Quorum-sensing control of biofilm factors in Staphylococcus epidermidis.
J. Infect. Dis. 188:706-718.

40. Yarwood, J.M., Bartels, D.J., Volper, E.M., and Greenberg, E.P. 2003 Expression of the accesory gene regulator quorum sensing system in biofilms of Staphylococcus aureus. ASM Conference on Biofilms 2003. Victoria, British Columbia, Canada. 39. (Abstr.)

41. Schwan, W.R., Langhorne, M.H., Ritchie, H.D., and Stover, C.K. 2003. Loss of hemolysin expression in Staphylococcus aureus agr mutants correlates with selective survival during mixed infections in murine abscesses and wounds. FEMS Immunol. Med. Microbiol. 38:23-28.

42. Papakyriacou, H., Vaz, D., Simor, A., Louie, M., and McGavin, M.J. 2000 Molecular analysis of the accessory gene regulator (agr) locus and balance of virulence factor expression in epidemic methicillin-resistant Staphylococcus aureus. J. Infect. Dis. 181:990-1000.

43. Somerville, G.A., et al. 2002. In vitro serial passage of Staphylococcus aureus: changes in physiology, virulence factor production, and agr nucleotide sequence. J. Bacteriol. 184:1430-1437. 